\title{
Multiobject Holographic Feedback Control of Differential Algebraic System with Application to Power System
}

\author{
Lanmei Cong, ${ }^{1,2}$ Xiaocong Li, ${ }^{1}$ and Ancai Zhang ${ }^{2}$ \\ ${ }^{1}$ College of Electrical Engineering, Guangxi University, Nanning, Guangxi 530005, China \\ ${ }^{2}$ School of Automobile Engineering, Linyi University, Linyi, Shandong 276005, China \\ Correspondence should be addressed to Lanmei Cong; lysyclm@126.com
}

Received 13 October 2014; Accepted 2 May 2015

Academic Editor: Hari M. Srivastava

Copyright (c) 2015 Lanmei Cong et al. This is an open access article distributed under the Creative Commons Attribution License, which permits unrestricted use, distribution, and reproduction in any medium, provided the original work is properly cited.

\begin{abstract}
A multiobject holographic feedback (MOHF) control method for studying the nonlinear differential algebraic (NDA) system is proposed. In this method, the nonlinear control law is designed in a homeomorphous linear space by means of constructing the multiobject equations (MOEq) which is in accord with Brunovsky normal form. The objective functions of MOEq are considered to be the errors between the output functions and their references. The relative degree for algebraic system is defined that is key to connecting the nonlinear and the linear control laws. Pole assignment method is addressed for the stability domain of this MOHF control. Since there is no any approximation, the MOHF control is effective in governing the dynamic performance stably both to the small and major disturbance. The application in single machine infinite system (SMIS) shows that this approach is effective in the improvement of stable and transient stability for power system on the disturbance of active power or three-phase short circuit fault.
\end{abstract}

\section{Introduction}

Power system shows strong nonlinear properties, especially in the action of large disturbance such as three-phase short circuit fault. Many effective control theories and methods have been proposed for this nonlinear system in literatures, such as differential geometry theory $[1,2]$, Lyapunov energy function $[3,4]$ and Hamiltonian function $[5,6]$, machine learning, and neural network [7-10]. Feedback linearization based on differential geometry theory is effective in the stability control of the nonlinear power system [2]. Different from the approximate linearization, Differential geometry method designs the nonlinear control law by constructing the mapping from the nonlinear space to the homeomorphous linear without approximation. Tan and Wang in [11] designed the adaptive excitation and phase shifter controller based on third-order generator model. Kennedy et al. in [12] designed a nonlinear excitation controller based on a form of state feedback linearization using the geometric approach. With the work of Sastry and Isidori [13], the adaptive control of "minimum-phase" nonlinear system was studied by state feedback exactly linearization and several initial results were derived.

Developing the differential geometry theory, Li et al. in [14] proposed the Multi-index Nonlinear Control (MINC) method. Their research demonstrated that it was not necessary for the system to satisfy the condition of completely exact linearization, which is usually too strict condition to satisfy for most of systems. Li et al. also addressed that the part exact linearization made the selection of output function more flexible to adapt the control requirement of the controlled system, which made MINC method get more satisfied control performance. Li et al. [14] also proposed an effective and practical method of determining output function that was linear combination of state vectors. The precondition of MINC method adopting part exact linearization is that the zero-dynamic must be stable, which is possibly difficult to prove for general system. For solving this problem, Liu et al. in [15] proposed the multiobject holographic feedback (MOHF) control method. This method designed the nonlinear feedback law in linear space by constructing multiobjective equations that satisfy Brunovsky normal form 
[16]. Furthermore, it is unnecessary to prove the stability of zero-dynamic. These research works are based on the ordinary differential equation (ODE) model.

For most of the complicated power system, it is insufficient that ODE describes wholly the dynamic activity, since the connecting nodes of power system have to be formulated in algebraic equations. The general power system, hence, is usually modeled as differential algebraic equation (DAE).

Several typical methods are described in literatures for studying the differential algebraic system and its application in power systems. Kurina and März in [17] addressed the linear quadratic optimal control problems with constraints described by general linear DAEs with variable coefficients. The optimal feedback control matrix can be obtained by suitably formulating a Riccati DAE system, which is similar to the classical example in which the constraints are described by explicit ODE system. Tsolas et al. in [18] presented the preserving structure model of general power system and suggested that the DA model could be replaced locally by an ODE description. They developed further some geometric characterizations of the region of attraction for a stable equilibrium point. The transient stability was studied in [19, 20] based on preserving structure model and the total energy function were shaped. Wang and Chen [21] presented the differential geometric method that used the preserved structure model for DA systems. The Lyapunov function was proposed in [22] for the stability research of multimachine power systems. The energy function presented in [23] as well as [18] showed some properties of Lyapunov function. Kautsky et al. [24] described the numerical method for determining robust solutions of pole assignment by state feedback. The robust pole assignment problem is also discussed in [24]. One may see the eigenstructure assignment approach of the state output feedback pole placement for $m$ input $p$ output in [25]. The pole assignment problems were proposed by [26-28] with projection and deflation methods together with optimization approach, respectively.

This paper explores the MOHF control described by the differential algebraic model. As an example of application in power system, the feedback law is designed for single machine infinite system. The structure is organized as follows. Section 2 presents a model of general SIMO differential algebraic system. Section 3 defines the $\Gamma$ derivative and relative degree for differential algebraic system. Section 4 proposes the MOHF design method and pole assignment approach for this design. Section 5 designs a practical power system based on the preserving structure as an application of the MOHF theory. Section 6 gives the conclusions.

\section{Differential Algebraic Model}

Consider the single-input multiout differential algebraic power system:

$$
\begin{aligned}
\dot{x} & =f(x, w)+g(x, w) u, \\
0 & =\rho(x, w), \\
y & =h(x, w) .
\end{aligned}
$$

The first differential equation describes the behaviors of dynamic components of power system. The second algebraic equation shows the relations of the voltage, active power, and (or) reactive power flows among the nodes. $x \in R^{n}$ is state vector. $w \in R^{p}$ is algebraic variable. $u \in R$ is the input of the plant. $y \in R^{m}$ is the output function which is decided by control performance. $f(\cdot), g(\cdot)$, and $\rho(\cdot)$ are the following mapped functions:

$$
\begin{array}{ll}
f: R^{n} \times R^{p} \longrightarrow R^{n} ; & (x, w) \longmapsto f(x, w), \\
g: R^{n} \times R^{p} \longrightarrow R^{n} ; & (x, w) \longmapsto g(x, w), \\
\rho: R^{n} \times R^{p} \longrightarrow R^{p} ; & (x, w) \longmapsto \rho(x, w) .
\end{array}
$$

The design in this paper is based on the following assumptions.

Assumption 1. $f(x, w), g(x, w), \rho(x, w)$, and $h(x, w)$ are smooth manifolds.

Assumption 2. $f(x, w), g(x, w)$, and $\rho(x, w)$ satisfy the compatible initial conditions, which are if the input $u=0$ there exist $f\left(x_{e}, w_{e}\right)=0, g\left(x_{e}, w_{e}\right)=0$, and $\rho\left(x_{e}, w_{e}\right)=0$ on the equilibrium point $\left(x_{e}, w_{e}\right)$ of (1). Without loss of generality, we assume that $\left(x_{e}, w_{e}\right)=(0,0)$.

Assumption 3. The Jacobi matrix of $\rho(x, w)$ with respect to $w$ is nonsingular.

\section{Relative Degree of the Differential Algebraic System}

The definition of the relative degree for ordinary differential equation system was given by Sastry and Isidori in [13]. We redefine it here in order to design differential algebraic system. Firstly, a special matrix is given as

$$
\Lambda(x, w)=\left[\begin{array}{c}
I_{n} \\
-\left[\frac{\partial \rho}{\partial w}\right]^{-1} \frac{\partial \rho}{\partial x}
\end{array}\right]
$$

where $I_{n}$ is an unit matrix of $n \times n$. This matrix $\Lambda(x, w)$ exists in the condition of Assumption 3 holding.

The definition of $\Gamma$ derivative is formulated as follows.

Definition 4. The product of the Jacobi matrix of output function $h(x, w)$ with respect to $x$ and $w$, the matrix $\Lambda(x, w)$, and the vector field $f(x, w)$ are defined as $\Gamma$ derivative of $h(x, w)$ with respect to $f(x, w)$ in differential algebraic system; that is

$$
\Gamma_{f} h(x, w)=\left[\begin{array}{ll}
\frac{\partial h(x, w)}{\partial x} & \frac{\partial h(x, w)}{\partial w}
\end{array}\right] \Lambda(x, w) f(x, w) .
$$

The higher $\Gamma$ derivatives are then defined as

$$
\begin{aligned}
& \Gamma_{f}^{2} h(x, w)=\Gamma_{f}\left(\Gamma_{f} h(x, w)\right) \\
& \quad=\left[\begin{array}{ll}
\frac{\partial\left(\Gamma_{f} h(x, w)\right)}{\partial x} & \frac{\partial\left(\Gamma_{f} h(x, w)\right)}{\partial w}
\end{array}\right] \Lambda(x, w)
\end{aligned}
$$




$$
\begin{aligned}
& \cdot f(x, w) \\
& \vdots \\
& \Gamma_{f}^{\lambda} h(x, w)=\Gamma_{f}\left(\Gamma_{f}^{\lambda-1} h(x, w)\right) \\
& \quad=\left[\frac{\partial\left(\Gamma_{f}^{\lambda-1} h(x, w)\right)}{\partial x} \frac{\partial\left(\Gamma_{f}^{\lambda-1} h(x, w)\right)}{\partial w}\right] \Lambda(x, w) \\
& \quad \cdot f(x, w) .
\end{aligned}
$$

Correspondingly, the $\Gamma$ derivative of the $\Gamma_{f}^{i} h(x, w)(i=$ $1,2, \ldots, \lambda)$ to $g(x, w)$ is defined as

$$
\begin{aligned}
& \Gamma_{g} \Gamma_{f} h(x, w) \\
& \quad=\left[\frac{\partial\left(\Gamma_{f} h(x, w)\right)}{\partial x} \frac{\partial\left(\Gamma_{f} h(x, w)\right)}{\partial w}\right] \Lambda(x, w) \\
& \cdot g(x, w) \\
& \vdots \\
& \Gamma_{g} \Gamma_{f}^{\lambda-1} h(x, w) \\
& \quad=\left[\frac{\partial\left(\Gamma_{f}^{\lambda-1} h(x, w)\right)}{\partial x} \frac{\partial\left(\Gamma_{f}^{\lambda-1} h(x, w)\right)}{\partial w}\right] \Lambda(x, w) \\
& \quad \cdot g(x, w) .
\end{aligned}
$$

An immediate definition of the relative degree for the differential algebraic systems is yielded as the following.

Definition 5. The DA system of (1) is said to have relative degree $\lambda$ to the output function $y=h(x, w)$, if and only if the following conditions holds:

$$
\begin{array}{ll}
\Gamma_{g} \Gamma_{f}^{i} h(x, w)=0 & i=1,2, \ldots, \lambda-2, \\
\Gamma_{g} \Gamma_{f}^{i} h(x, w) \neq 0 & i=\lambda-1 .
\end{array}
$$

With these definitions of $\Gamma$ derivative and relative degree, we can establish the homeomorphous mapping between nonlinear space and linear in the MOHF control design of next section.

\section{MOHF Control Design}

In this section, we describe the MOHF design method in detail. We establish, firstly, the adaptive model and then discuss the rules of choosing objective function. In the following section we propose the structure of multiobjective equation and design feedback control law. The problem of pole assignment is solved in Section 4.4.

4.1. Adaptive Model. Consider the reference model:

$$
\begin{aligned}
\dot{x}_{r} & =f\left(x_{r}, w_{r}\right)+g\left(x_{r}, w_{r}\right) u_{r}, \\
0 & =\rho\left(x_{r}, w_{r}\right) .
\end{aligned}
$$

The adaptive model is derived from (9) and (1):

$$
\begin{aligned}
(\Delta x)^{\prime} & =\Delta f(x, w)+g(x, w) \Delta u, \\
0 & =\Delta \rho(x, w),
\end{aligned}
$$

where

$$
\begin{aligned}
(\Delta x)^{\prime} & =\dot{x}-\dot{x}_{r}, \\
\Delta u & =u-u_{r}, \\
\Delta f & =f(x, w)-f\left(x_{r}, w_{r}\right), \\
\Delta \rho & =\rho(x, w)-\rho\left(x_{r}, w_{r}\right) .
\end{aligned}
$$

The reference adaptive output function of plant (1) is followed as

$$
\Delta y=y-y_{r}=h(x, w)-h\left(x_{r}, w_{r}\right),
$$

where $y_{r}=h\left(x_{r}, w_{r}\right)$ is the output of (9) and also the reference output of plant (1).

4.2. Objective Functions. Objective functions are the variables that need to be constrained in control. These functions are considered as the form

$$
I=\left[\begin{array}{llll}
I_{1} & I_{2} & \cdots & I_{m}
\end{array}\right]^{T},
$$

where

$$
I_{i}=y_{i}-y_{i r}=\Delta y_{i} \quad(i=1,2, \ldots, m)
$$

and $y_{i}$ and $y_{i r}$ are the $i$ th component of output function in (12) and its reference trajectory.

In this MOHF control, the output functions (12) are determined for the purpose of getting satisfied control object. They include not only the practical outputs of the system but also other variables related to control properties. We consider them as the linear combination of state vectors and algebraic variables, which means

$$
\mathbf{y}=\mathbf{h}(x, w)=\mathbf{c}_{x} \mathbf{x}+\mathbf{c}_{w} \mathbf{w},
$$

where $y \in R^{m}$, and

$$
\mathbf{c}_{x}=\left[\begin{array}{cccc}
c_{x 11} & c_{x 12} & \cdots & c_{x 1 n} \\
c_{x 21} & c_{x 22} & \cdots & c_{x 2 n} \\
\vdots & \vdots & \cdots & \vdots \\
c_{x m 1} & c_{x m 2} & \cdots & c_{x m n}
\end{array}\right],
$$




$$
\mathbf{c}_{w}=\left[\begin{array}{cccc}
c_{w 11} & c_{w 12} & \cdots & c_{w 1 n} \\
c_{w 21} & c_{w 22} & \cdots & c_{w 2 n} \\
\vdots & \vdots & \cdots & \vdots \\
c_{w m 1} & c_{w m 2} & \cdots & c_{w m n}
\end{array}\right]
$$

are the constant matrices decided by the dynamic and stability properties of plant.

Remark 6. The choice of objective function acts importantly in MOHF control. It decides the effect of control and the complexity of feedback law. Two factors fall into main considerations. The first, also the most important, is the relations between objective functions and control performance. The second is the practicability of the designed control law, since the excessively complicated law is probably difficult to carry out and maybe declines the robustness. Based on both of the considerations, it is of high priority to let the relative degree hold

$$
\lambda=1
$$

in determining the objective functions. Compared with higher $\lambda$, this can make feedback law more simple and hence easy to be used in practical system. For most of system, (17) is not a hard condition if the objective functions are arranged appropriately. Our following design is based on this condition satisfied.

4.3. Design of MOHF Control Law. In this section, we use the objective functions in (13) to construct the multiobjective equation that is in agreement with Brunovsky normal form [16] and design the nonlinear feedback law in linear space. The multiobjective equation is formed as

$$
\dot{I}=A I+B v
$$

where

$$
A=\left[\begin{array}{ccccc}
0 & 1 & \cdots & 0 & 0 \\
0 & 0 & \cdots & 0 & 0 \\
\vdots & \vdots & \cdots & \vdots & \vdots \\
0 & 0 & \cdots & 0 & 1 \\
0 & 0 & \cdots & 0 & 0
\end{array}\right]
$$

$$
B=\left[\begin{array}{c}
0 \\
0 \\
\vdots \\
0 \\
1
\end{array}\right]
$$

are Brunovsky constant matrices.

This system (18) is called the extra system. The feedback law $v$ is called extra system control law. It is designed as the following form according to linear optimal theory

$$
\begin{aligned}
v & =\dot{I}_{m}=-k_{1} I_{1}-k_{2} I_{2}-\cdots-k_{m} I_{m} \\
& =-k_{1} \Delta y_{1}-k_{2} \Delta y_{2}-\cdots-k_{m} \Delta y_{m} .
\end{aligned}
$$

The feedback matrices $\mathbf{K}=\left[\begin{array}{llll}-k_{1} & -k_{2} & \cdots & -k_{q}\end{array}\right\rfloor$ can be yielded by the next pole assignment.

The control law $u$ of system (1) is accordingly called as the inner system control law, which is bridged with $v$ by (5)-(7). From (18) we have

$$
\begin{aligned}
v & =\dot{I}_{m}=\dot{y}_{m}-\dot{y}_{m r} \\
& =\Gamma_{F f} h_{m}(x, w)+\Gamma_{F g} \Gamma_{F f}^{0} h_{m}(x, w) u-\dot{y}_{m r}
\end{aligned}
$$

and from (20), the inner feedback control law $\Delta u$ is obtained:

$$
\Delta u=\frac{\left(v+\dot{y}_{m r}\right)}{\Gamma_{F g} \Gamma_{F f}^{0} h_{m}(x, w)}=\frac{\left[-k_{1}\left(y_{1}-y_{1 r}\right)-k_{2}\left(y_{2}-y_{2 r}\right)-\cdots-k_{m}\left(y_{m}-y_{m r}\right)-\Gamma_{F f} h_{m}(x, w)+\dot{y}_{m r}\right]}{\Gamma_{F g} \Gamma_{F f}^{0} h_{m}(x, w)} .
$$

Remark 7. This MOHF method uses the extra control law $v$ to govern the objective functions to their equilibriums. The homeomorphous transformations of (21)-(22) transfer this law to equivalent input $\Delta u$ of nonlinear system to constrain the nonlinear dynamic oscillation. Since this transformation from nonlinear linear space to linear holds all the state information without approximation, this approach may enhance the dynamic stability when $v$ is designed appropriately.

The feedback and output constant matrix are designed by the following pole assignment.

4.4. Pole Assignment. The linear reference system of (1) is derived from the Taylor expansion on $\left(x_{r}, w_{r}\right)$

$$
\begin{aligned}
\dot{x}_{o} & \\
= & f\left(x_{r}, w_{r}\right)+f_{x}\left(x_{r}, w_{r}\right)\left(x-x_{r}\right) \\
& +f_{w}\left(x_{r}, w_{r}\right)\left(w-w_{r}\right)+g\left(x_{r}, w_{r}\right) u_{r} \\
& +\left[g_{x}\left(x_{r}, w_{r}\right)\left(x-x_{r}\right)+g_{w}\left(x_{r}, w_{r}\right)\left(w-w_{r}\right)\right] u
\end{aligned}
$$

0

$$
\begin{aligned}
= & \rho_{r}\left(x_{r}, w_{r}\right)+\rho_{r}\left(x_{r}, w_{r}\right)\left(x-x_{r}\right) \\
& +\rho_{w}\left(x_{r}, w_{r}\right)\left(w-w_{r}\right) .
\end{aligned}
$$


The linear expansion of output function (2) is

$$
\begin{aligned}
y= & h\left(x_{r}, w_{r}\right)+h_{x}\left(x_{r}, w_{r}\right)\left(x-x_{r}\right) \\
& +h_{w}\left(x_{r}, w_{r}\right)\left(w-w_{r}\right),
\end{aligned}
$$

where $f_{x}(\cdot), f_{w}(\cdot), \rho_{x}(\cdot), \rho_{w}(\cdot), h_{x}(\cdot), h_{w}(\cdot)$ are Jacobian matrices.

For the stability control of power system, the aim of control is to make the system reinstate to its original equilibrium or achieve a new equilibrium after the disturbance deleted. So we consider that the reference trajectories of (23)-(25) are equilibrium; that is

$$
\begin{aligned}
& \dot{x}_{r}=f\left(x_{e}, w_{e}\right)+g\left(x_{e}, w_{e}\right) u_{e}, \\
& \rho_{r}=\rho\left(x_{e}, w_{e}\right), \\
& y_{r}=h\left(x_{e}, w_{e}\right) .
\end{aligned}
$$

Substituting (24) into (23) and (25), the linear adaptive model is as follows:

$$
\begin{aligned}
\hat{\dot{x}}-\widehat{\dot{x}}_{o r} & =\widehat{A}\left(\widehat{x}-\widehat{x}_{r}\right)+\widehat{B}\left(u-u_{r}\right), \\
\widehat{y}-\widehat{y}_{r} & =\widehat{C}\left(\widehat{x}-\widehat{x}_{r}\right)
\end{aligned}
$$

which is expressed concisely as

$$
\begin{aligned}
& \Delta \widehat{\dot{x}}=\widehat{A} \Delta \widehat{x}+\widehat{B} \Delta u, \\
& \Delta \widehat{y}=\widehat{C} \hat{x} .
\end{aligned}
$$

Selecting the nonsingular linear transformation matrix $T_{c}$, let $\Delta \hat{x}_{c}=\widehat{x}_{c}-\widehat{x}_{c r}=T_{c} \Delta \hat{x}$; then (29) are transformed to the following controllable normal form

$$
\begin{aligned}
& \Delta \dot{\hat{x}}_{c}=\widehat{A}_{c} \Delta \widehat{x}_{c}+\widehat{B}_{c} \Delta u, \\
& \Delta \widehat{y}_{c}=\widehat{C}_{c} \Delta \widehat{x}_{c},
\end{aligned}
$$

where

$$
\begin{aligned}
& \widehat{A}_{c}=T_{c} \widehat{A} T_{c}^{-1}=\left[\begin{array}{ccccc}
0 & 1 & 0 & \cdots & 0 \\
0 & 0 & 1 & \cdots & 0 \\
\vdots & \vdots & \vdots & \vdots & \vdots \\
0 & 0 & 0 & \cdots & 1 \\
-a_{n} & -a_{n-1} & -a_{n-2} & \cdots & -a_{1}
\end{array}\right]_{n \times n} \\
& \widehat{B}_{c}=T_{c} \widehat{B}=\left[\begin{array}{lllll}
0 & 0 & \cdots & 0 & 1
\end{array}\right]^{T} \text {, } \\
& \widehat{C}_{c}=\widehat{C} T_{c}^{-1}=\left[\begin{array}{llll}
\widehat{C}_{c 1} & \widehat{C}_{c 2} & \cdots & \widehat{C}_{c m}
\end{array}\right]^{T} .
\end{aligned}
$$

From (29), (30), and (31) we have that

$$
\dot{I}_{m}=\frac{\partial \Delta \widehat{y}_{c m}}{\partial \Delta \widehat{x}_{c}} \Delta \dot{\hat{x}}_{c}=\widehat{C}_{c m} A_{c} \Delta \widehat{x}_{c}+\widehat{C}_{c m} \widehat{B}_{c} \Delta u .
$$

Assume that (30)-(31) hold condition (17), we get the feedback law

$$
\begin{aligned}
\Delta u & =\left(\widehat{C}_{c m} \widehat{B}_{c}\right)^{-1} \\
\cdot & {\left[-k_{c 1}\left(I_{1}-I_{1 r}\right)-\cdots-k_{c m}\left(I_{m}-I_{m r}\right)-\widehat{C}_{c m} \widehat{A}_{c}\right] } \\
& \cdot\left(\widehat{x}_{c}-\widehat{x}_{c r}\right) .
\end{aligned}
$$

Substituting (34) into (30) we get

$$
\begin{aligned}
\Delta \widehat{x}_{c} & =\left[\widehat{A}_{c}+\widehat{B}_{c}\left(\widehat{C}_{c m} \widehat{B}_{c}\right)^{-1}\right. \\
\cdot & \left.\left(-k_{c 1} \widehat{C}_{c 1}-\cdots-k_{c m} \widehat{C}_{c m}-\widehat{C}_{c m} \widehat{A}_{c}\right)\right] \cdot\left(\widehat{x}_{c}-\widehat{x}_{c r}\right) \\
& =\widetilde{A}_{c} \Delta \widehat{x}_{c},
\end{aligned}
$$

where

$$
\begin{gathered}
\widetilde{A}_{c}=\widehat{A}_{c}+\widehat{B}_{c} \widehat{K}_{c}=\left[\begin{array}{cccc}
0 & 1 & \cdots & 0 \\
\vdots & \vdots & \cdots & \vdots \\
0 & 0 & \cdots & 1 \\
-\widetilde{k}_{c 1} & -\widetilde{k}_{c 2} & \cdots & -\widetilde{k}_{c n}
\end{array}\right] \\
\widetilde{k}_{c 1}=\frac{k_{c 1} \widehat{c}_{c 11}+\cdots+k_{c m} \widehat{c}_{c m 1}}{\widehat{c}_{c m, n}}
\end{gathered}
$$

The characteristic polynomial of (31) is

$$
f(\lambda)=\lambda^{n}+\widetilde{k}_{c 1} \lambda^{n-1}+\cdots+\widetilde{k}_{c(n-1)} \lambda+\widetilde{k}_{c n}
$$

Remark 8. Equation (27) describes the linear asymptotical system of plant (1) in the neighborhood of hyperbolic singular point. According to Hartman-Grobman theory, the above pole assignment to the linear system can achieve locally stability control of (1).

Remark 9. This MOHF design method supplies an inner adaptive reference track that is the equilibrium state. In case that the feedback law constrains the objective functions to its reference tracks, the stability of the system is held.

\section{Application}

As an example of application, we designed MOHF law for single machine infinite system (SMIS) based on a DA model. 
The DA model of the SMIS is

$$
\begin{aligned}
\dot{E}_{q i}^{\prime} & =-\frac{x_{d \sum}}{T_{d o}^{\prime} x_{d \Sigma}^{\prime}} E_{q}^{\prime}-\frac{1}{T_{d o}^{\prime}} \frac{x_{d \Sigma}-x_{d \Sigma}^{\prime}}{x_{d \Sigma}^{\prime}} V^{2} \cos \delta \\
& +\frac{1}{T_{d o}^{\prime}} E_{q e} \\
\dot{\delta} & =(\omega-1) \omega_{0} \\
\dot{\omega} & =\frac{1}{T_{J}}\left(P_{m}-P_{e}\right)-\frac{D}{T_{J}}(\omega-1), \\
0 & =V_{G} \\
& -\sqrt{\left(\frac{x_{q}}{x_{q \Sigma}} V \sin \delta\right)^{2}+\left(\frac{x_{d}^{\prime}}{x_{d \Sigma}^{\prime}} V \cos \delta+\frac{x_{e}}{x_{d \Sigma}^{\prime}} E_{q}^{\prime}\right)^{2}}, \\
0 & =P_{e}-\frac{1}{x_{d \Sigma}^{\prime}} E_{q}^{\prime} V \sin \delta-\frac{x_{d \Sigma}^{\prime}-x_{q \Sigma}}{2 x_{d \Sigma}^{\prime} x_{q \Sigma}} V^{2} \sin (2 \delta),
\end{aligned}
$$

where $x=\left[\begin{array}{lll}\delta & \omega & E_{q}^{\prime}\end{array}\right]^{T}$ is the state vector of the generator and $w=\left[\begin{array}{ll}V_{G} & P_{e}\end{array}\right]^{T}$ the algebraic constraint variable. $\delta$ is the rotor angle, $\omega$ is the rotor speed, and $E_{q}^{\prime}$ is the transient internal voltage of the generator. $x_{d}$ and $x_{q}$ are direct-axis and quadrature-axis synchronous reactance of the generator, respectively. $x_{d}^{\prime}$ is direct-axis transient reactance. $x_{d \Sigma}=x_{d}+$ $x_{e}+x_{l}, x_{d \sum}^{\prime}=x_{d}^{\prime}+x_{e}+x_{l}$, and $x_{q \sum}=x_{q}+x_{e}+x_{l}$, where, $x_{e}$ is the transformer reactance and $x_{l}$ the transmission line reactance. $V_{G}$ is output terminal voltage of the generator and $V$ is the voltage at infinite bus. $T_{d o}^{\prime}$ is the time constant of the field-winding with open-circuit armature winding. $D$ is the constant of damper winding and $T_{J}$ the inertia time constant of the rotor. are

Compared with (1), the matrices $f(\cdot), g(\cdot)$, and $\rho(\cdot)$ of (38)

$$
\begin{aligned}
& f(x, w) \\
& =\left[\begin{array}{c}
-\frac{x_{d \Sigma}}{T_{d o}^{\prime} x_{d \Sigma}^{\prime}} E_{q}^{\prime}-\frac{1}{T_{d o}^{\prime}} \frac{x_{d \Sigma}-x_{d \Sigma}^{\prime}}{x_{d \Sigma}^{\prime}} V^{2} \cos \delta+\frac{1}{T_{d o}^{\prime}} E_{q e} \\
(\omega-1) \omega_{0} \\
\frac{1}{T_{J}}\left(P_{m}-P_{e}\right)-\frac{D}{T_{J}}(\omega-1)
\end{array}\right], \\
& g(x, w)=\left[\begin{array}{lll}
\frac{1}{T_{d o}^{\prime}} & 0 & 0
\end{array}\right]^{T}, \\
& \rho(x, w) \\
& =\left[\begin{array}{l}
\rho_{1}(x, w) \\
\rho_{2}(x, w)
\end{array}\right] \\
& =\left[\begin{array}{c}
V_{G}-\sqrt{\left(\frac{x_{q}}{x_{q \Sigma}} V \sin \delta\right)^{2}+\left(\frac{x_{d}^{\prime}}{x_{d \Sigma}^{\prime}} V \cos \delta+\frac{x_{e}}{x_{d \Sigma}^{\prime}} E_{q}^{\prime}\right)^{2}} \\
P_{e}-\frac{1}{x_{d \Sigma}^{\prime}} E_{q}^{\prime} V \sin \delta-\frac{x_{d \Sigma}^{\prime}-x_{q \Sigma}}{2 x_{d \Sigma}^{\prime} x_{q \Sigma}} V^{2} \sin (2 \delta)
\end{array}\right] .
\end{aligned}
$$

as

$$
\begin{aligned}
& y_{1}=c_{1} \omega, \\
& y_{2}=c_{2} E_{q}^{\prime}, \\
& y_{3}=c_{3} V_{g}, \\
& y_{1 r}=c_{1} \omega_{r}, \\
& y_{2 r}=c_{2} E_{q r}^{\prime}, \\
& y_{3 r}=c_{3} V_{g r} .
\end{aligned}
$$

The objective function is as follows:

$$
\begin{aligned}
& I_{1}=y_{1}-y_{1 r}=c_{1}\left(\omega-\omega_{r}\right)=c_{1} \Delta \omega, \\
& I_{2}=y_{2}-y_{2 r}=c_{2}\left(E_{q}^{\prime}-E_{q r}^{\prime}\right)=c_{2} \Delta E_{q}^{\prime}, \\
& I_{3}=y_{3}-y_{3 r}=c_{3}\left(V_{g}-\Delta V_{r}\right)=c_{3} \Delta V_{g} .
\end{aligned}
$$

Construct the multiobjective equation in accordance with the Brunovsky form as

$$
\left[\begin{array}{l}
\dot{I}_{1} \\
\dot{I}_{2} \\
\dot{I}_{3}
\end{array}\right]=\left[\begin{array}{lll}
0 & 1 & 0 \\
0 & 0 & 1 \\
0 & 0 & 0
\end{array}\right]\left[\begin{array}{l}
I_{1} \\
I_{2} \\
I_{3}
\end{array}\right]+\left[\begin{array}{l}
0 \\
0 \\
1
\end{array}\right] v .
$$

The feedback law

$$
\begin{aligned}
v & =-k_{1} I_{1}-k_{2} I_{2}-k_{3} I_{3} \\
& =-k_{1}\left(c_{1} \Delta \omega\right)-k_{2}\left(c_{2} \Delta E_{q}^{\prime}\right)-k_{3}\left(c_{3} \Delta V_{g}\right),
\end{aligned}
$$

where $\mathbf{K}=\left[\begin{array}{lll}-k_{1} & -k_{2} & -k_{3}\end{array}\right]$ and the constants $c_{1}, c_{2}$, and $c_{3}$ are designed according to pole assignment.

From (43) (44), we get another expression of $v$

$$
v=\dot{I}_{3}=\dot{h}_{3}(x, w)=c_{3} \Delta \dot{V}_{g} .
$$

From (39)-(40), substituting (5) (7) in (46), we have that

$$
\begin{aligned}
v & =\dot{h}_{3}(x, w)=c_{3} \Delta \dot{V}_{g}=c_{3}\left(\dot{V}_{g}-\dot{V}_{g r}\right)=\Gamma_{f} h_{3}(x, w) \\
& +\Gamma_{g} \Gamma_{f}^{0} h_{3}(x, w) u \\
& =\left[\begin{array}{ll}
\frac{\partial h_{3}(x, w)}{\partial x} & \frac{\partial h_{3}(x, w)}{\partial w}
\end{array}\right] \Lambda(x, w) \Delta f(x, w) \\
& +\left[\frac{\partial\left(\Gamma_{f}^{0} h_{3}(x, w)\right)}{\partial x} \frac{\partial\left(\Gamma_{f}^{0} h_{3}(x, w)\right)}{\partial w}\right] \Lambda(x, w) \\
& \cdot g(x, w) \Delta\left(u-u_{r}\right),
\end{aligned}
$$

where $\Delta f(x, w)=f(x, w)-f\left(x_{r}, w_{r}\right)$ and $\Delta u=u-u_{r}\left(u_{r}\right.$ is the input of the SIMS in the stable state). From (46) we have

$$
\left[\begin{array}{ll}
\frac{\partial h_{3}(x, w)}{\partial x} & \frac{\partial h_{3}(x, w)}{\partial w}
\end{array}\right]=\left[\begin{array}{lllll}
0 & 0 & 0 & c_{3} & 0
\end{array}\right]
$$



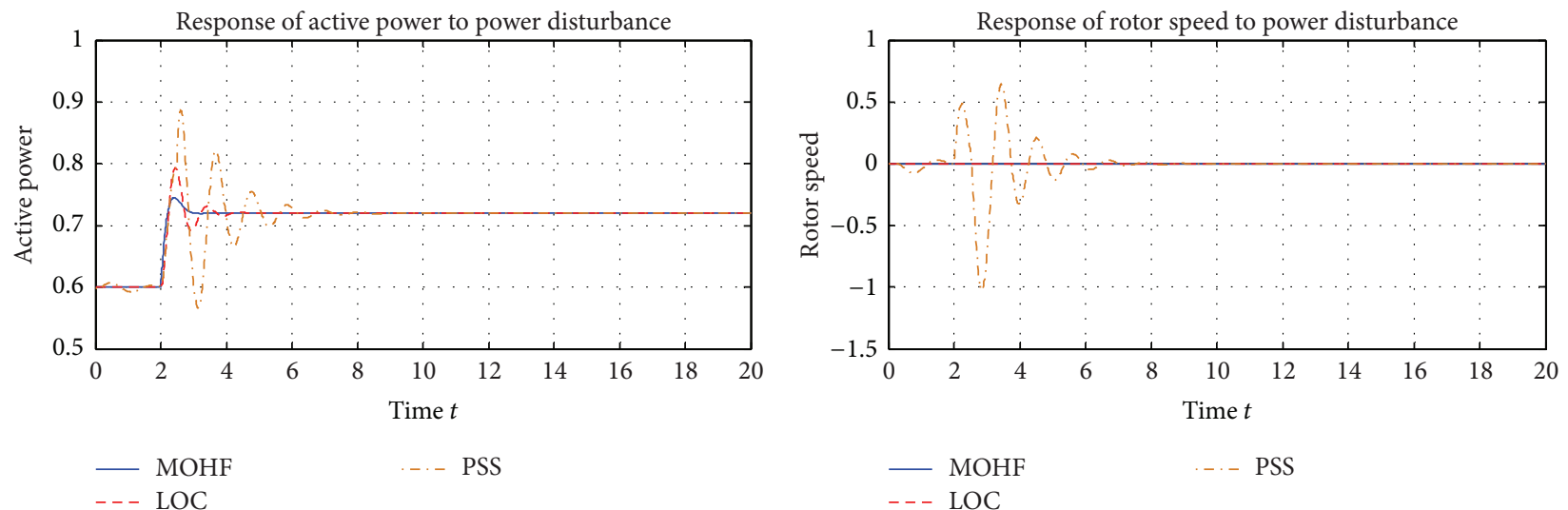

(a) The curve of $P_{e}$

(b) The curve of $\Delta \omega$

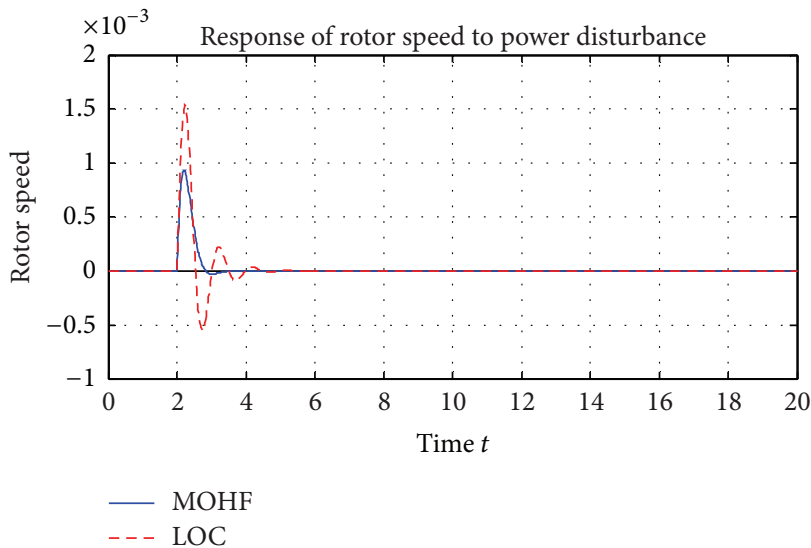

(c) The curve of $\Delta \omega$ for MOHF control compared to LOC

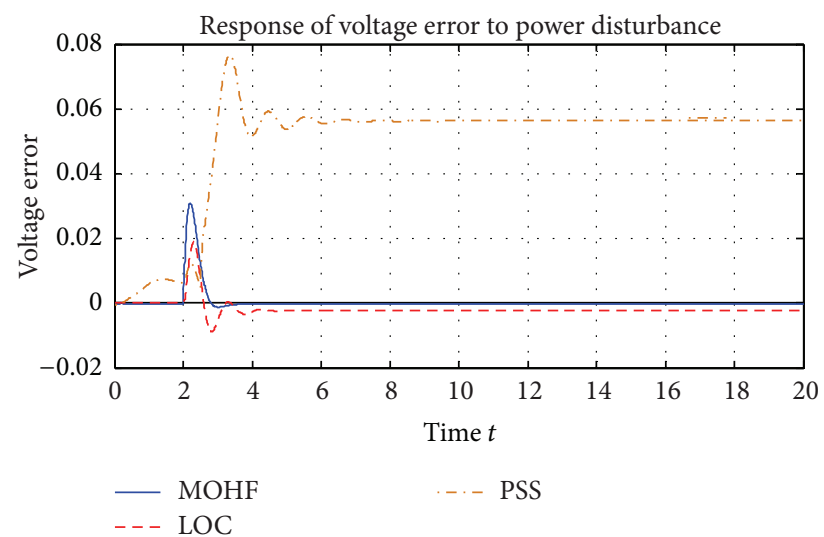

(d) The curve of $\Delta V_{g}$

FIGURE 1: System responses to the disturbance of the mechanical power stepping up 20\% regulation.

From $\rho(x, w)$ of $(40)$ we have that

$$
\begin{aligned}
-\left[\frac{\partial \rho}{\partial w}\right]^{-1} \frac{\partial \rho}{\partial x} & =-\left[\begin{array}{ll}
\frac{\partial \rho_{1}}{\partial V_{g}} & \frac{\partial \rho_{1}}{\partial P_{e}} \\
\frac{\partial \rho_{2}}{\partial V_{g}} & \frac{\partial \rho_{2}}{\partial P_{e}}
\end{array}\right]\left[\begin{array}{lll}
\frac{\partial \rho_{1}}{\partial E_{q}^{\prime}} & \frac{\partial \rho_{1}}{\partial \delta} & 0 \\
\frac{\partial \rho_{2}}{\partial E_{q}^{\prime}} & \frac{\partial \rho_{2}}{\partial \delta} & 0
\end{array}\right] \\
& =\left[\begin{array}{ll}
1 & 0 \\
0 & 1
\end{array}\right]\left[\begin{array}{lll}
\frac{\partial \rho_{1}}{\partial E_{q}^{\prime}} & \frac{\partial \rho_{1}}{\partial \delta} & 0 \\
\frac{\partial \rho_{2}}{\partial E_{q}^{\prime}} & \frac{\partial \rho_{2}}{\partial \delta} & 0
\end{array}\right] \\
& =\left[\begin{array}{lll}
\frac{\partial \rho_{1}}{\partial E_{q}^{\prime}} & \frac{\partial \rho_{1}}{\partial \delta} & 0 \\
\frac{\partial \rho_{2}}{\partial E_{q}^{\prime}} & \frac{\partial \rho_{2}}{\partial \delta} & 0
\end{array}\right] .
\end{aligned}
$$

The $\Lambda(x, w)$ is gotten by the substitution (49) into (4)

$$
\Lambda(x, w)=\left[\begin{array}{c}
I_{n} \\
-\left[\frac{\partial \rho}{\partial w}\right]^{-1} \frac{\partial \rho}{\partial x}
\end{array}\right]
$$

$$
=\left[\begin{array}{ccccc}
1 & 0 & 0 & \frac{\partial \rho_{1}}{\partial E_{q}^{\prime}} & \frac{\partial \rho_{1}}{\partial \delta} \\
0 & 1 & 0 & \frac{\partial \rho_{2}}{\partial E_{q}^{\prime}} & \frac{\partial \rho_{2}}{\partial \delta} \\
0 & 0 & 1 & 0 & 0
\end{array}\right]^{T}
$$

Combining (48)-(50) with (5)-(7), the $\Gamma_{f} h_{3}(x, w)$ and $\Gamma_{g} \Gamma_{f}^{0} h_{3}(x, w)$ are as follows:

$$
\Gamma_{f} h_{3}(x, w)
$$$$
=\left[\begin{array}{lllll}
0 & 0 & 0 & c_{3} & 0
\end{array}\right]\left[\begin{array}{ccccc}
1 & 0 & 0 & \frac{\partial \rho_{1}}{\partial E_{q}^{\prime}} & \frac{\partial \rho_{2}}{\partial E_{q}^{\prime}} \\
0 & 1 & 0 & \frac{\partial \rho_{1}}{\partial \delta} & \frac{\partial \rho_{2}}{\partial \delta} \\
0 & 0 & 1 & 0 & 0
\end{array}\right]^{T} \Delta f(x, w)
$$$$
=\left[\begin{array}{ccc}
c_{3} \frac{\partial \rho_{1}}{\partial E_{q}^{\prime}} & c_{3} \frac{\partial \rho_{1}}{\partial \delta} & 0
\end{array}\right] \Delta f(x, w),
$$

$\Gamma_{g} \Gamma_{f}^{0} h_{3}(x, w)$ 


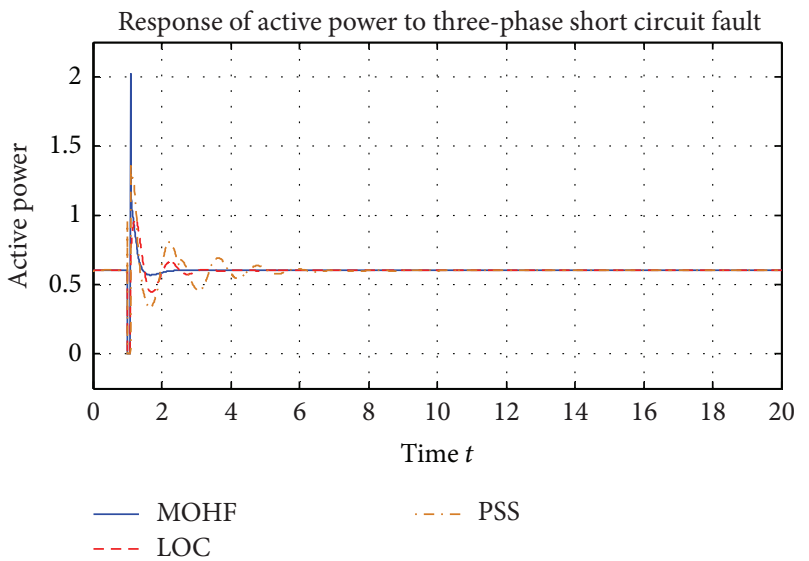

(a) The curve of $P_{e}$

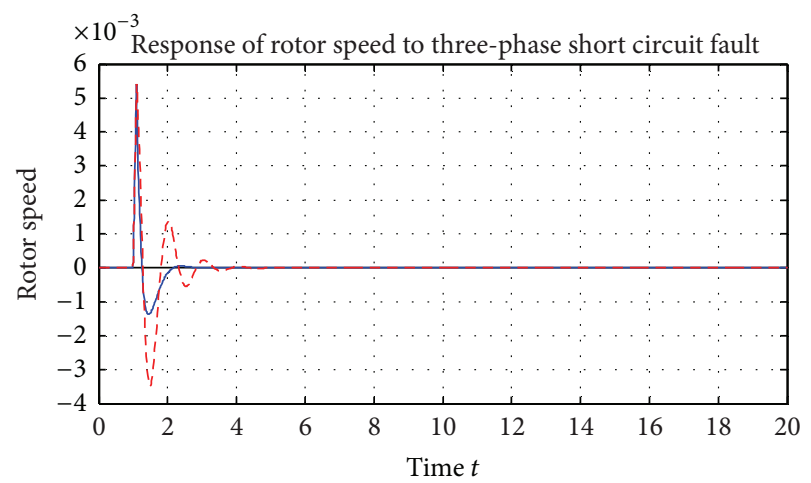

- MOHF

-. LOC

(c) The curve of $\Delta \omega$ for MOHF control compared to LOC

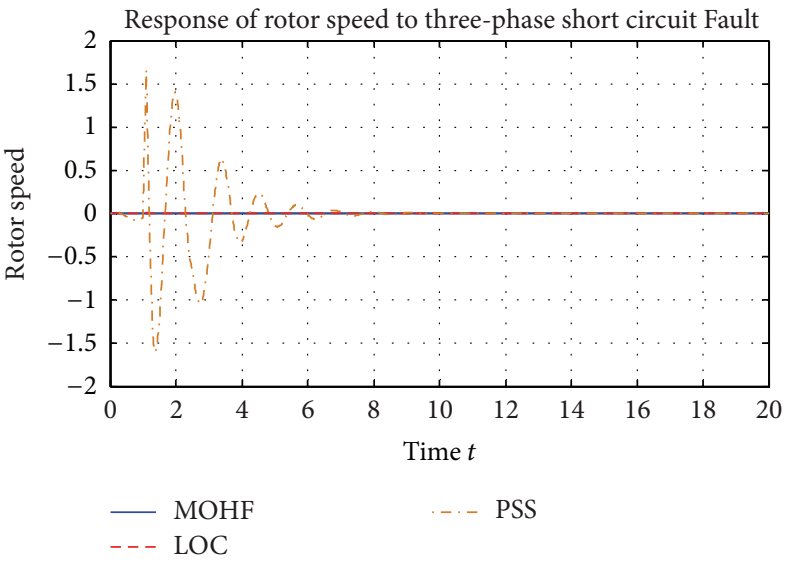

(b) The curve of $\Delta \omega$

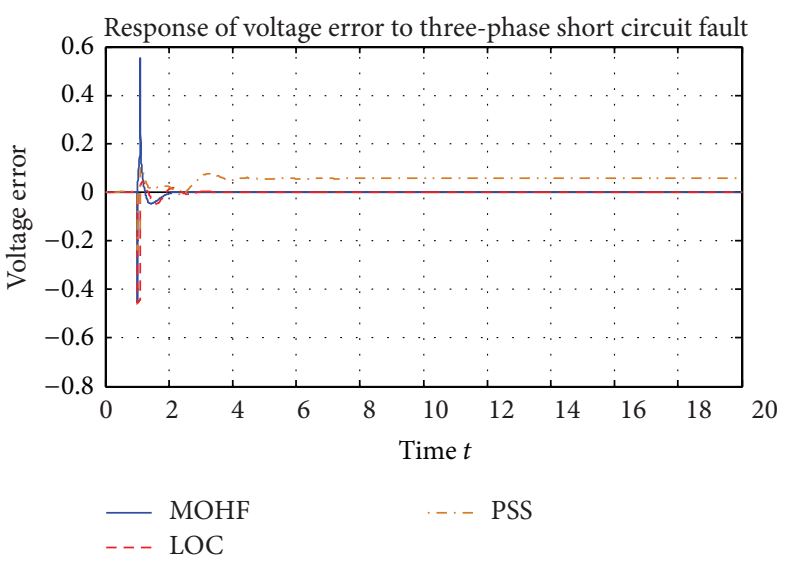

(d) The curve of $\Delta V_{g}$

FIGURE 2: System responses to the disturbance of a three-phase short circuit fault.

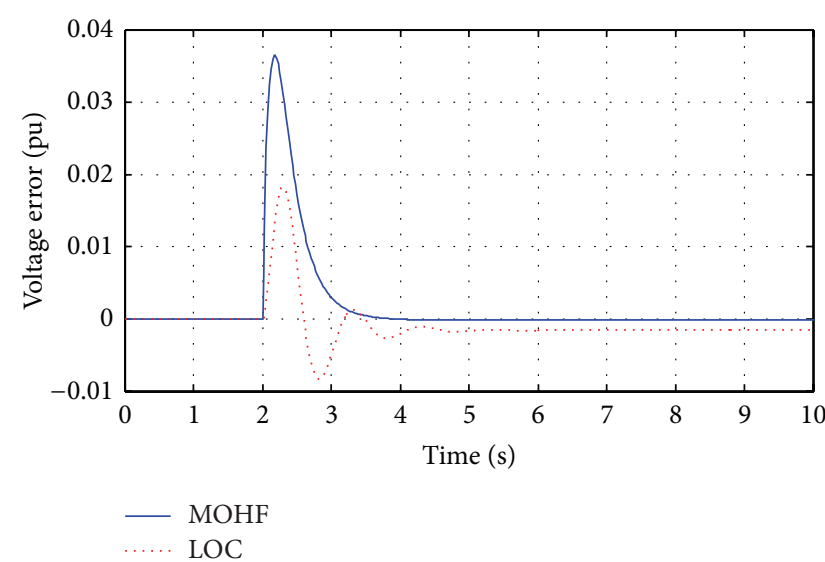

(a) The curve of $\Delta V_{g}$

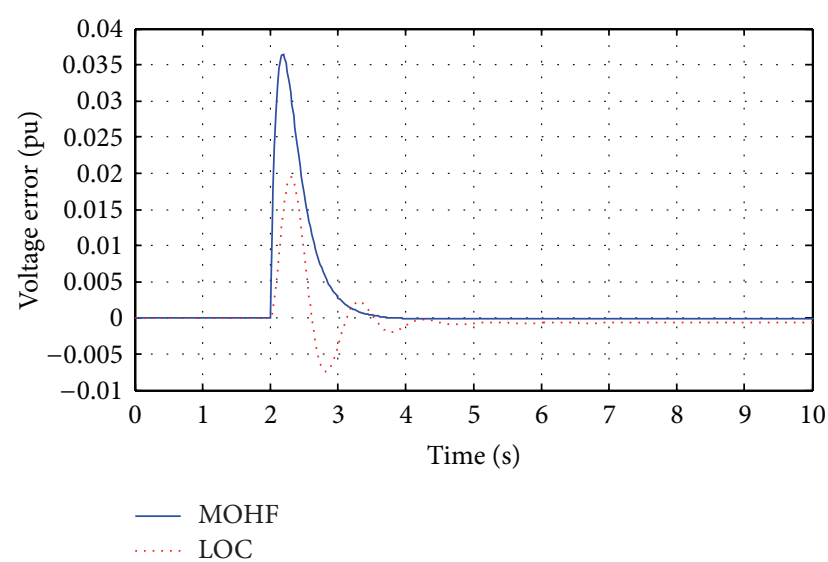

(b) The curve of $\Delta V_{g}$

FIGURE 3: System responses to adjustment of the feedback matrix. 


$$
\begin{aligned}
& =\left[\begin{array}{lllll}
0 & 0 & 0 & c_{3} & 0
\end{array}\right]\left[\begin{array}{ccccc}
1 & 0 & 0 & \frac{\partial \rho_{1}}{\partial E_{q}^{\prime}} & \frac{\partial \rho_{2}}{\partial E_{q}^{\prime}} \\
0 & 1 & 0 & \frac{\partial \rho_{1}}{\partial \delta} & \frac{\partial \rho_{2}}{\partial \delta} \\
0 & 0 & 1 & 0 & 0
\end{array}\right]^{T} g(x, w) \\
& =\left[\begin{array}{lll}
c_{3} \frac{\partial \rho_{1}}{\partial E_{q}^{\prime}} & c_{3} \frac{\partial \rho_{1}}{\partial \delta} & 0
\end{array}\right] g(x, w) \neq 0 .
\end{aligned}
$$

The nonlinear adaptive feedback control law is derived as

$$
\Delta u=\frac{\left(v-\Gamma_{f} h_{3}(x, w)\right)}{\Gamma_{g} \Gamma_{f}^{0} h_{3}(x, w)} .
$$

Simulation and Discussions. The constants of (51)-(52) are given as $x_{d}=x_{q}=1.962, x_{d}^{\prime}=0.246, T_{d o}^{\prime}=7.4, T_{J}=11$, $D=0.5$, and $x_{e}=0.2$. The initial running conditions are considered as $V_{G 0}=1.0, P_{0}=0.6$, and $Q_{0}=0.0798$.

Figure 1 shows the simulation results when the disturbance of the active power steps up 20\% with the constants $c_{1}=500, c_{2}=0$, and $c_{3}=-10 ; k_{1}=k_{2}=k_{3}=150$ for MOHF.

In Figure 1, the solid lines show the simulink results of MOHF control; the dotted lines are the results of linear optimal control (LOC), and the dash-dotted lines depict the dynamics of adjustment when the power system stabilizer (PSS) is implemented in the power system. As the overshoot of rotor speed $\Delta \omega$ of PSS is much larger than both of the MOHF control and LOC, additional plot (Figure 1(c)) is used to illustrate the dynamics precisely.

Figure 1(a) shows the response of the electromagnetic power $P_{e}$ of the generator when the disturbance of mechanical power imposed on the SMIS. From these simulink curves one can see that when the system is exerted by a constant disturbance from the active power, the MOHF method can adjust the rotor angle by supporting an equivalent input $\Delta u$ to improve the electromagnetic power and attain rapidly new power balance with good stable and dynamic properties. This dynamic process is accompanied with smaller oscillation and overshoot than LOC and PSS method, which is beneficial for protecting the generator against the mechanical shock. Figure 1(b) is the response of rotor speed whose precise comparison of MOHF and LOC is shown in Figure 1(c). Oscillations of small magnitude and low frequency may present limitations on power transfer capability in some cases and affect the stability [29, 30]. Figures 1(b) and 1(c) demonstrate that the MOHF control is more effective in damping frequency oscillations of system. Figure $1(\mathrm{~d})$ is the plot of the generator terminal voltage deviation. It gives the result that the MOHF control method adjusts the output voltage to stable state in very short time with zero error. Although the overshoot is bigger than LOC, it contents the power system criterion. Figures 1(b), 1(c), and 1(d) show that with the control of MOHF, the system attains better electric qualities in regards to voltage fluctuation and low frequency oscillation than the LOC and PSS when this system is subjected to an active power disturbance.
Figure 2 shows the responses when the three-phase short circuit fault occurs at one second in the infinite bus of SIMS and is solved after 0.1 second.

The dynamics of $P_{e}, \Delta \omega$, and $\Delta V_{g}$ are showed in Figures 2(a), 2(b), 2(c), and 2(d), respectively, with the solid blue lines for MOHF, red dotted lines for LOC, and orange dash-dotted lines for PSS. From Figure 2(a) one can see that, when the serious fault acts on the system, the dynamic feedback law $\Delta u$ of MOHF can effectively restrain the oscillation of power and make the active power $P_{e}$ rapidly reinstate its balance point. Figure 2(b) and Figure 2(c) show that the dynamic response of rotor speed for MOHF holds smaller maximum overshoot, shorter settling time, and fewer frequency oscillation than LOC and PSS. From Figure 2(d) one can see that the dynamic reactive power compensation of the MOHF ensures the output voltage of the generator with good dynamic and stable performance. It is clear from Figure 2 that the MOHF controls the dynamic behavior of system in serious disturbance more effectively than LOC and PSS.

Robust Analysis. Figures 3(a) and 3(b) are the responses while the control constants are adjusted. The solid lines show the results of the MOHF control and the dotted lines are results of the LOC.

This figure (Figure 3(a)) shows the simulation result with the output constant $c_{3}$ of MOHF changed from $c_{3}=-15$ of Figure 2 to this figure's $c_{3}=-10$, other constants keep no change, and the feedback matrix $\mathbf{K}$ of LOC is adjusted from $K=\left[\begin{array}{lll}75 & 10 & -2000\end{array}\right]$ to $K=\left[\begin{array}{lll}70 & 10 & -2000\end{array}\right]$. Figure 3(b) shows the result of constants $c_{1}$ and $K$ from $c_{1}=500$ to $c_{1}=505$ and $K$ from $K=\left[\begin{array}{lll}70 & 10 & -2000\end{array}\right]$ to $K=$ $\left[\begin{array}{lll}70 & 12 & -2000\end{array}\right]$.

From these figures one may find that when the control constant matrices are adjusted, the MOHF still contains good transient response with zero error while the LOC with error. This result indicates that the MOHF method holds better robustness than LOC.

\section{Conclusion}

This paper has explored the principles and design method of multiobject holographic feedback control for nonlinear differential algebraic system and applied them to design the feedback law of single machine infinite bus power system. The concept of relative degree and $\Gamma$ derivative were defined which are used to connect the output function and equivalent input. Based on these works, the homeomorphous mapping from nonlinear space to linear was established. By constructing the multiobject equation which is in accordance with Brunovsky normal form, the control objects of nonlinear system were transformed to linear space to implement control. Pole assignment is used to design the output constant matrix to attain the stability.

Since this transformation from nonlinear space to linear employed no approximation and held all of the nonlinear information, this MOHF control may inhibit the oscillation more effectively than LOC and PSS approaches while the small or large disturbance imposes on the system. The practical application in the SMIS demonstrates the good 
effect of this inhibition. Unnecessary proving the stability of zero-dynamics, the MOHF control based on differential algebraic model may be helpful in the research of more general electrical power system and other similar nonlinear differential algebraic systems.

\section{Conflict of Interests}

The authors declare that there is no conflict of interests regarding the publication of this paper.

\section{Acknowledgment}

This paper is funded by the National Natural Science Foundation of China (Nos. 51267001 and 61304023), Guangxi scientific research and technical development project (1412200629) and Guangxi Natural Science Foundation (2014GXNSFAA118338).

\section{References}

[1] A. Isidori, Nonlinear Control Systems, Communications and Control Engineering Series, Springer, New York, NY, USA, 3rd edition, 1995.

[2] Q. Lu and Y. Z. Sun, "Nonlinear stabilizing control of multimachine systems," IEEE Transactions on Power Systems, vol. 4, no. 1, pp. 236-241, 1989.

[3] H. Liu, Z. Hu, and Y. Song, "Lyapunov-based decentralized excitation control for global asymptotic stability and voltage regulation of multi-machine power systems," IEEE Transactions on Power Systems, vol. 27, no. 4, pp. 2262-2270, 2012.

[4] V. Azbe and R. Mihalic, "The control strategy for an IPFC based on the energy function," IEEE Transactions on Power Systems, vol. 23, no. 4, pp. 1662-1669, 2008.

[5] Y. Z. Sun, Q. J. Liu, Y. H. Song, and T. L. Shen, "Hamiltonian modelling and nonlinear disturbance attenuation control of TCSC for improving power system stability," IEEE Transactions on Power Systems, vol. 23, no. 4, pp. 1662-1669, 2008.

[6] Y. Wang, D. Cheng, C. Li, and Y. Ge, "Dissipative Hamiltonian realization and energy-based $L_{2}$-disturbance attenuation control of multi-machine power systems," IEEE Transactions on Automatic Control, vol. 48, no. 8, pp. 1428-1433, 2003.

[7] Y. Jin and B. Sendhoff, "Pareto-based multiobjective machine learning: an overview and case studies," IEEE Transactions on Systems, Man and Cybernetics Part C: Applications and Reviews, vol. 38, no. 3, pp. 397-415, 2008.

[8] R. Hadidi and B. Jeyasurya, "Reinforcement learning approach for controlling power system stabilizers," Canadian Journal of Electrical and Computer Engineering, vol. 34, no. 3, Article ID 5443857, pp. 99-103, 2009.

[9] M. Farahani, “A multi-objective power system stabilizer," IEEE Transactions on Power Systems, vol. 28, no. 3, pp. 2700-2707, 2013.

[10] G. K. Venayagamoorthy and R. G. Harley, "A continually online trained neurocontroller for excitation and turbine control of a turbogenerator," IEEE Transactions on Energy Conversion, vol. 16, no. 3, pp. 261-269, 2001.

[11] Y. L. Tan and Y. Wang, "Nonlinear excitation and phase shifter controller for transient stability enhancement of power systems using adaptive control law," International Journal of Electrical Power and Energy Systems, vol. 18, no. 6, pp. 397-403, 1996.
[12] D. Kennedy, D. Miller, and V. Quintana, "A nonlinear geometric approach to power system excitation control and stabilization," International Journal of Electrical Power and Energy Systems, vol. 20, no. 8, pp. 501-515, 1998.

[13] S. S. Sastry and A. Isidori, "Adaptive control of linearizable systems," IEEE Transactions on Automatic Control, vol. 34, no. 11, pp. 1123-1131, 1989.

[14] X. C. Li, S. J. Cheng, H. Wei, and S. R. Wang, "Multi-index nonlinear integrated control for turbine generator unit," Proceedings of the Chinese Society of Electrical Engineering, vol. 23, no. 4, pp. 96-101, 2003.

[15] H. Liu, X.-C. Li, and H. Wei, "Nonlinear excitation control for generator unit based on NCOHF," Proceedings of the Chinese Society of Electrical Engineering, vol. 27, no. 1, pp. 14-18, 2007.

[16] R. B. Gardner and W. F. Shadwick, "The GS algorithm for exact linearization to Brunovsky normal form," IEEE Transactions on Automatic Control, vol. 37, no. 2, pp. 224-230, 1992.

[17] G. A. Kurina and R. März, "Feedback solutions of optimal control problems with DAE constraints," SIAM Journal on Control and Optimization, vol. 46, no. 4, pp. 1277-1298, 2007.

[18] N. A. Tsolas, A. Arapostathis, and P. P. Varaiya, "A structure preserving energy function for power system transient stability analysis," IEEE Transactions on Circuits and Systems, vol. 32, no. 10, pp. 1041-1049, 1985.

[19] B. He, X. Zhang, and X. Zhao, "Transient stabilization of structure preserving power systems with excitation control via energy-shaping," International Journal of Electrical Power and Energy Systems, vol. 29, no. 10, pp. 822-830, 2007.

[20] J. Arifa, S. Rayb, and B. Chaudhuria, “Transient stabilization of structure preserving power systems with excitation control via energy-shaping," International Journal of Electric Power and Energy System, vol. 45, no. 1, pp. 87-97, 2013.

[21] J. Wang and C. Chen, "Nonlinear control of differential algebraic model in power systems," Proceedings of the Chinese Society of Electrical Engineering, vol. 21, no. 8, pp. 15-23, 2001.

[22] A. R. Bergen, D. J. Hill, and C. L. de Marcot, "Lyapunov function for multimachine power systems with generator flux decay and voltage dependent loads," International Journal of Electrical Power and Energy Systems, vol. 8, no. 1, pp. 2-10, 1986.

[23] N. Narasimhamurthi and M. T. Musavi, "A generalized energy function for transient stability analysis of power systems," IEEE Transactions on Circuits and Systems, vol. 31, no. 7, pp. 637-645, 1984.

[24] J. Kautsky, N. K. Nichols, and P. van Dooren, "Robust pole assignment in linear state feedback," International Journal of Control, vol. 41, no. 5, pp. 1129-1155, 1985.

[25] A. Varga, "Robust and minimum norm pole assignment with periodic state feedback," IEEE Transactions on Automatic Control, vol. 45, no. 5, pp. 1017-1022, 2000.

[26] O. Bachelier, J. Bosche, and D. Mehdi, "On pole placement via eigenstructure assignment approach," IEEE Transactions on Automatic Control, vol. 51, no. 9, pp. 1554-1558, 2006.

[27] Y. Saad, "Projection and deflation methods for partial pole assignment in linear state feedback," IEEE Transactions on Automatic Control, vol. 33, no. 3, pp. 290-297, 1988.

[28] S. Datta, D. Chakraborty, and B. Chaudhuri, "Partial pole placement with controller optimization," IEEE Transactions on Automatic Control, vol. 57, no. 4, pp. 1051-1056, 2012.

[29] E. V. Larsen and D. A. Swann, "Applying power system stabilizers part I: general concepts," IEEE transactions on power apparatus and systems, vol. 100, no. 6, pp. 3017-3024, 1981. 
[30] E. V. Larsen and D. A. Swann, "Applying power system stabilizers part II: performance objectives and tuning concepts," IEEE Transactions on Power Apparatus and Systems, vol. 100, no. 6, pp. 3025-3033, 1981. 


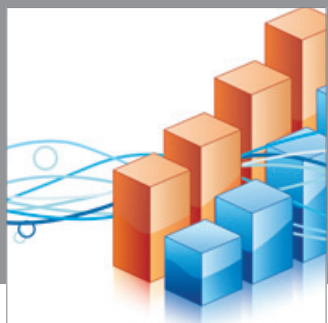

Advances in

Operations Research

mansans

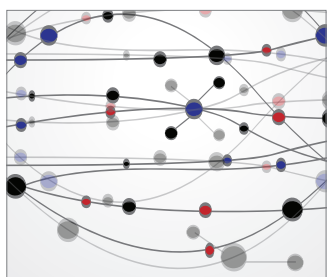

The Scientific World Journal
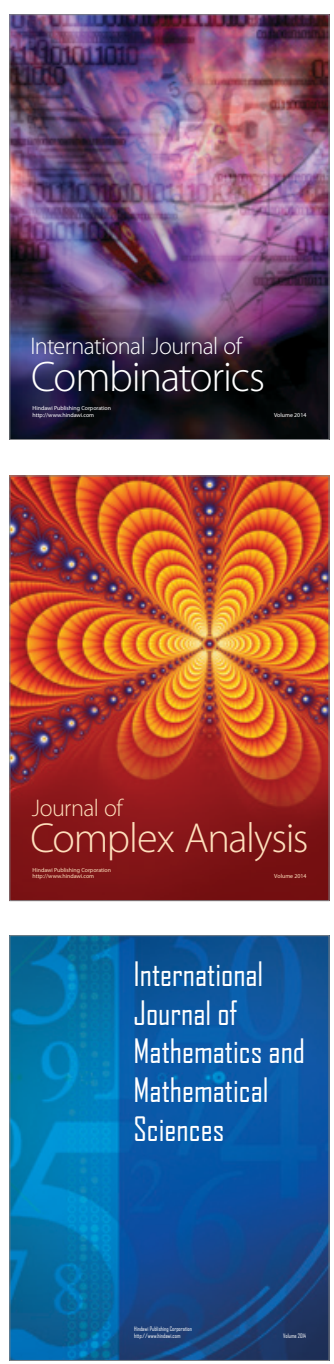
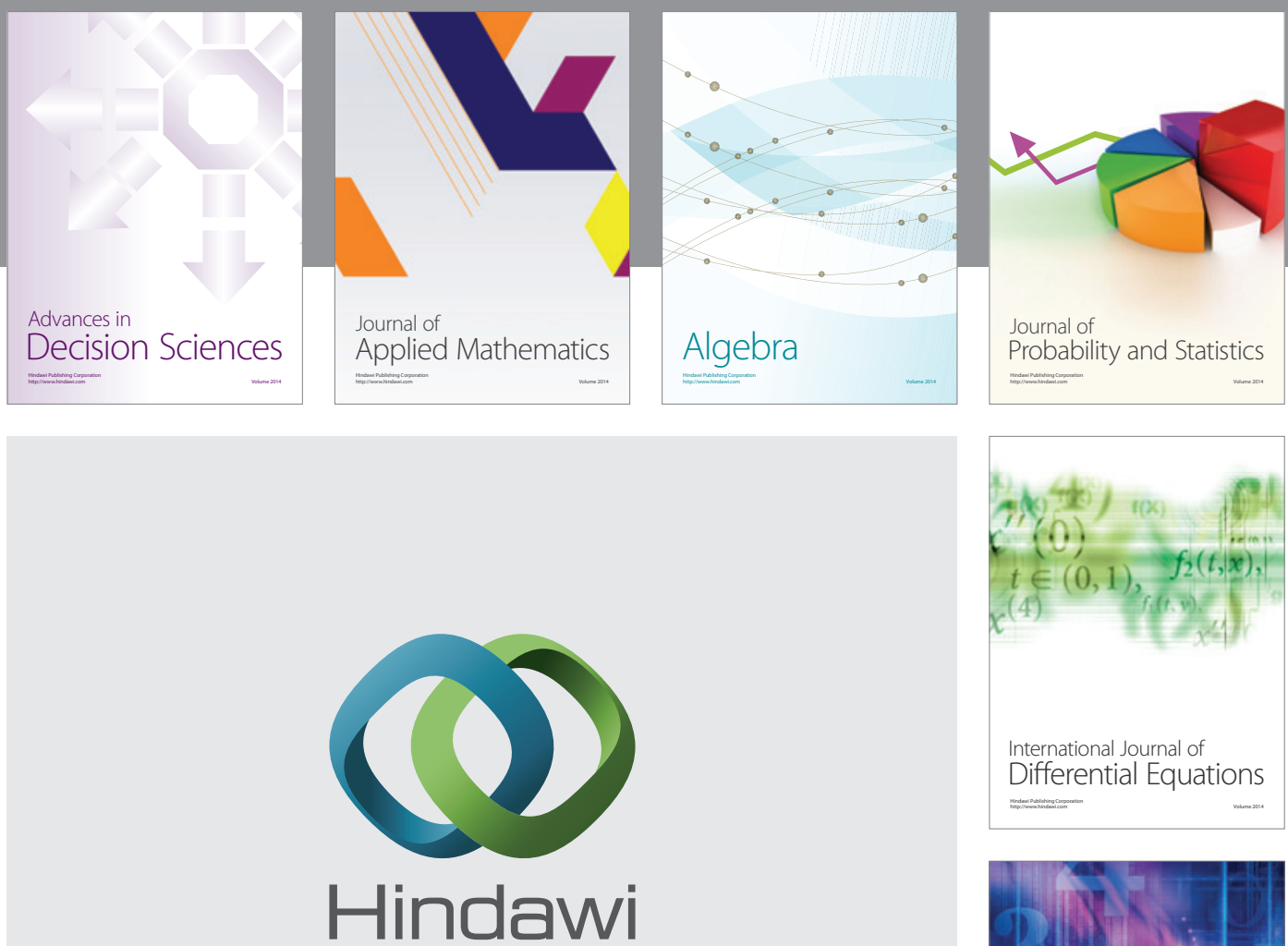

Submit your manuscripts at http://www.hindawi.com
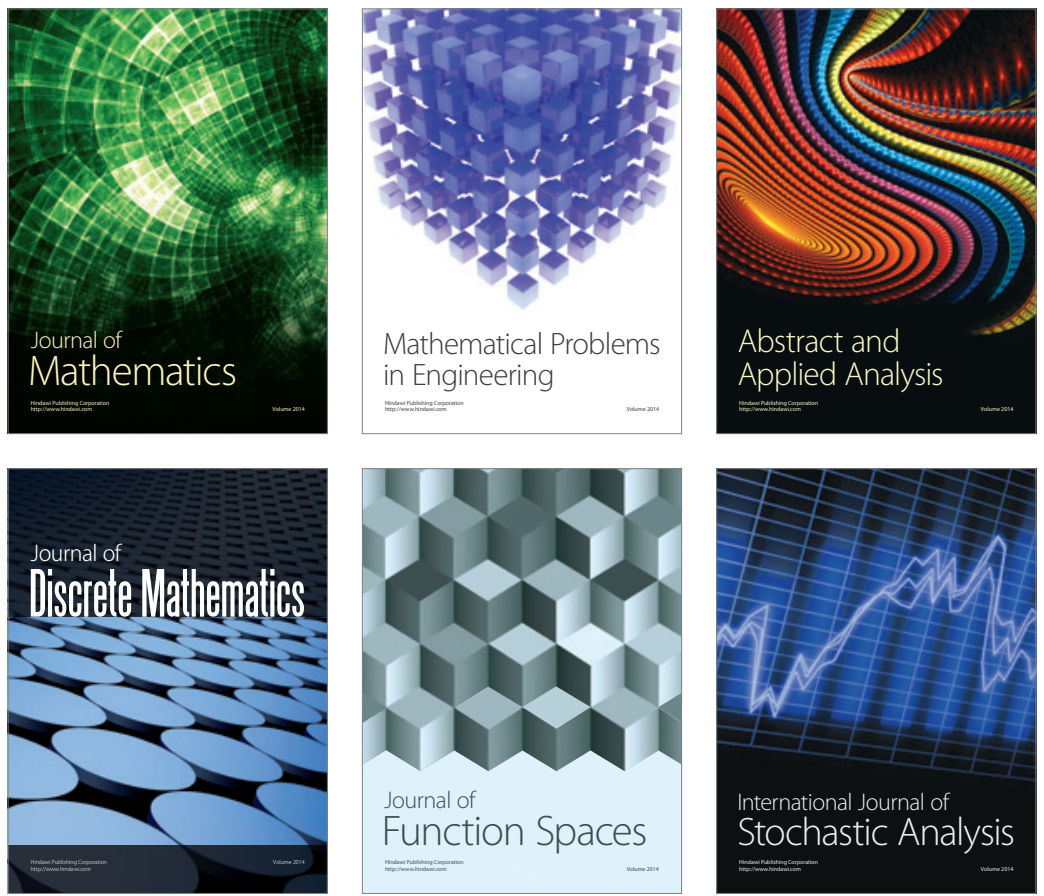

Journal of

Function Spaces

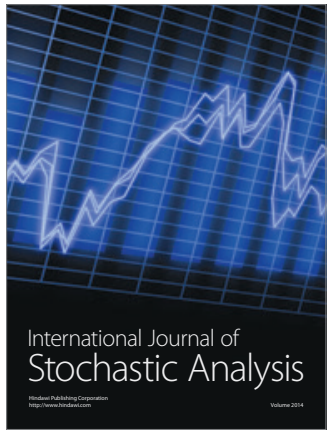

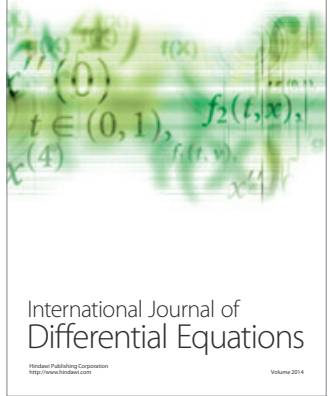
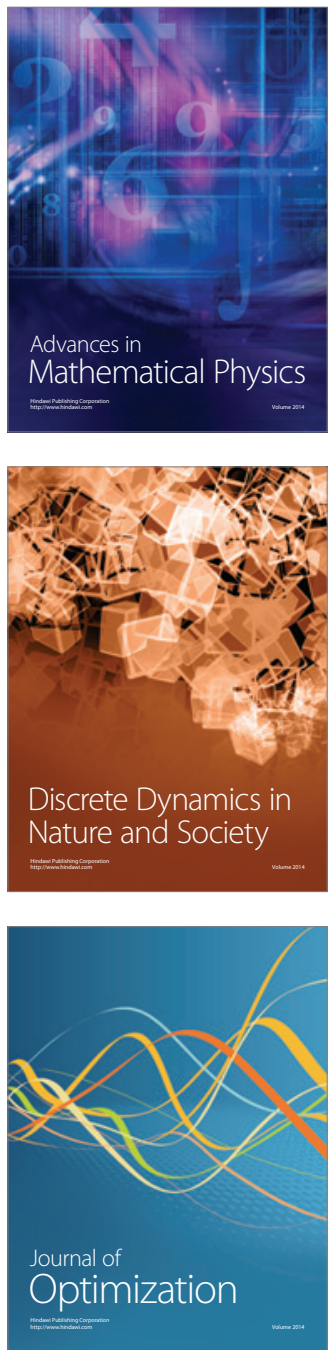